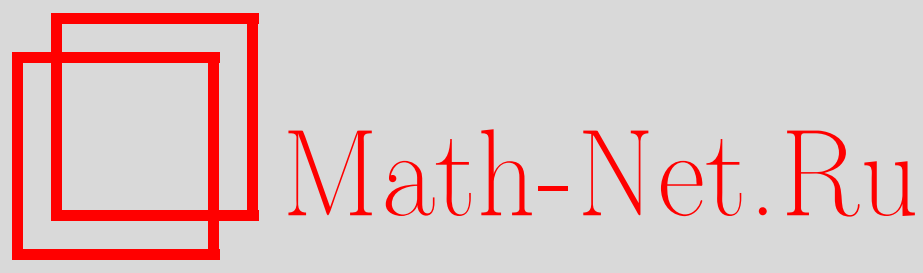

А. Бенхассин, Гамильтоновы системы дробного порядка с локально заданными потенциалами, ТМФ, 2018, том 195, номер 1, 81-90

DOI: https://doi.org/10.4213/tmf9371

Использование Общероссийского математического портала Math-Net.Ru подразумевает, что вы прочитали и согласны с пользовательским соглашением http://www.mathnet.ru/rus/agreement

Параметры загрузки:

IP : 44.207 .124 .84

26 апреля 2023 г., 16:25:44

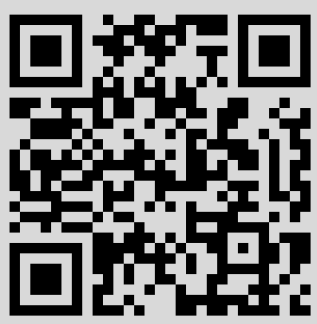




\section{ГАМИЛЬТОНОВЫ СИСТЕМЫ ДРОБНОГО ПОРЯДКА С ЛОКАЛЬНО ЗАДАННЫМИ ПОТЕНЦИАЛАМИ}

Изучаются решения следующей непериодической гамильтоновой системы дробного порядка $\alpha \in(1 / 2,1]$ :

$$
{ }_{-}{ }_{t} D_{\infty}^{\alpha}\left({ }_{-\infty} D_{t}^{\alpha} x(t)\right)-L(t) x(t)+\nabla W(t, x(t))=0, \quad x \in H^{\alpha}\left(\mathbb{R}, \mathbb{R}^{N}\right),
$$

где матрица $L(t) \in C\left(\mathbb{R}, \mathbb{R}^{N^{2}}\right), t \in \mathbb{R}, \mathrm{a}_{-\infty} D_{t}^{\alpha}$ и $_{t} D_{\infty}^{\alpha}-$ левая и правая дробные производные Лиувилля-Вейля порядка $\alpha$ на всей оси $\mathbb{R}$. С помощью симметричной теоремы о горном переходе доказано существование бесконечного числа решений системы в случае, когда матрица $L(t)$ не обязательно является коэрцитивной или равномерно положительно определенной, а потенциал $W(t, x)$ задан только локально в окрестности начала координат $x=0$. Доказанные теоремы значительно обобщают и улучшают ранее полученные результаты. Также приведены некоторые иллюстративные примеры.

Ключевые слова: дробные гамильтоновы системы, теория критической точки, симметричная теорема о горном переходе.

DOI: https://doi.org/10.4213/tmf9371

\section{1. ВВЕДЕНИЕ И ФОРМУЛИРОВКА ОСНОВНЫХ РЕЗУЛЬТАТОВ}

Гамильтоновы системы составляют важную часть нелинейного функционального анализа, поскольку они возникают при описании явлений в различных областях науки, таких как физика, астрономия, химия, биология, инженерные дисциплины и др. C тех пор как Ньютон записал уравнения, описывающие движение планет, и получил в качестве их решения эллиптические орбиты Кеплера, сложное динамическое поведение гамильтоновых систем стало привлекать внимание математиков и физиков. Вариационные методы исследования гамильтоновых систем впервые были использованы Пуанкаре, который применил принцип минимального действия в форме Якоби для изучения замкнутых орбит консервативной системы с двумя степенями свободы. В работе [1] Амброзетти и Рабинович доказали теорему о горном переходе, теорему о седловой точке, теорему о связанных множествах (linking theorem) и ряд

${ }^{*}$ Department of Mathematics, Higher Institute of Informatics and Mathematics, Monastir, Tunisia. E-mail: ab.hassine@yahoo.com 
чрезвычайно важных минимаксных форм теоремы о критической точке. Применение теории критической точки позволило сделать значительный шаг вперед в изучении гамильтоновых систем. Рабиновичем в работе [2] теорема о критической точке была впервые использована для доказательства существования периодических решений гамильтоновой системы первого порядка, а первый результат о множественности орбит был получен Амброзетти и Зелати [3]. С тех пор появилось большое количество публикаций по использованию теории критической точки и вариационных методов для доказательства существования гомо- и гетероклинических орбит гамильтоновых систем (см., например, работы [4], [5] и ссылки в них).

С другой стороны, в последнее десятилетие все возрастающую популярность и значимость при описании процессов с длинной памятью приобретает дробное исчисление. За деталями мы отсылаем к монографиям [6], [7] и ссылкам в них. Теория критической точки стала эффективным инструментом изучения проблемы существования решений дифференциальных уравнений дробного порядка с помощью построения дробных вариационных структур.

Недавно в работе [8] было показано, что теория критической точки является действенным подходом к решению проблемы существования решения следующей краевой задачи дробного порядка:

$$
{ }_{t} D_{T}^{\alpha}\left({ }_{0} D_{t}^{\alpha} x(t)\right)=\nabla W(t, x(t)) \quad \text { для почти всех } t \in[0, T], \quad x(0)=x(T),
$$

где $\alpha \in(1 / 2,1), x \in \mathbb{R}^{N}, W \in C^{1}\left([0, T] \times \mathbb{R}^{N}, \mathbb{R}\right)$. Было доказано, что задача имеет по крайней мере одно нетривиальное решение. В продолжение этой работы Торрес в работе [9] рассматривал следующую гамильтонову систему дробного порядка:

$$
-{ }_{t} D_{\infty}^{\alpha}\left({ }_{-\infty} D_{t}^{\alpha} x(t)\right)-L(t) x(t)+\nabla W(t, x(t))=0, \quad x \in H^{\alpha}\left(\mathbb{R}, \mathbb{R}^{N}\right),
$$

где $\alpha \in(1 / 2,1)$, матрица $L(t) \in C\left(\mathbb{R}, \mathbb{R}^{N^{2}}\right)$, функция $W$ принадлежит пространству $C^{1}\left(\mathbb{R} \times \mathbb{R}^{N}, \mathbb{R}\right)$ и $\nabla W(t, x)$ - ее градиент по переменной $x$. С помощью теоремы о горном переходе в работе [9] было показано, что задача (1.1) имеет по крайней мере одно нетривиальное решение. При этом предполагалось, что $L$ и $W$ удовлетворяют следующим условиям ${ }^{1)}$ :

$(\mathrm{L} 1)$ матрица $L(t) \in C\left(\mathbb{R}, \mathbb{R}^{N^{2}}\right)$ симметрична и положительно определена при всех значениях $t \in \mathbb{R}$;

(L2) наименьшее собственное значение матрицы $L(t)$ стремится к бесконечности при $t \rightarrow \infty$;

$(\mathrm{W} 1)$ равномерно по $t \in \mathbb{R}$ имеет место асимптотика $|\nabla W(t, x)|=o(|x|)$ при $|x| \rightarrow 0$;

(W2) существует функция $\bar{W} \in C\left(\mathbb{R}^{N}, \mathbb{R}\right)$ такая, что для всех $(t, x) \in \mathbb{R} \times \mathbb{R}^{N}$

$$
|W(t, x)|+|\nabla W(t, x)| \leqslant|\bar{W}(x)|
$$

$(\mathrm{AR})$ найдется постоянная $\mu>2$ такая, что для всех $t \in \mathbb{R}$ и $x \in \mathbb{R}^{N} \backslash\{0\}$

$$
0<\mu W(t, x) \leqslant \nabla W(t, x) \cdot x
$$

1) Всюду ниже точка в выражениях типа $x \cdot y$ обозначает скалярное произведение в $\mathbb{R}^{N}$ и $|x|-$ соответствующая норма элемента $x \in \mathbb{R}^{N}$. 
Мотивированные упомянутыми выше работами, мы изучаем вопрос о существовании бесконечно большого количества решений задачи (1.1), полагая, что $L$ не обязательно является коэрцитивной и равномерно положительно определенной матрицей, а потенциал $W$ задан только локально в окрестности начала координат $x=0$. Более точно, мы налагаем следующие условия:

(L3) существует постоянная $a_{0}>0$ такая, что для

$$
l(t):=\min _{x \in \mathbb{R}^{N},|x|=1} L(t) x \cdot x
$$

при всех $t \in \mathbb{R}$ выполнено неравенство $l(t)+a_{0} \geqslant 1$ и, кроме того, $\int_{\mathbb{R}} \frac{d t}{l(t)+a_{0}}<\infty$;

(W3) функция $W \in C^{1}\left(\mathbb{R} \times B_{\rho}(0), \mathbb{R}\right)$ четная, имеет место тождество $W(t, 0) \equiv 0$ и существует постоянная $c>0$ такая, что для всех $(t, x) \in \mathbb{R} \times B_{\rho}(0)$, где $B_{\rho}(0)-$ открытый шар в $\mathbb{R}^{N}$ с центром в нуле и радиусом $\rho$, справедлива оценка

$$
|\nabla W(t, x)| \leqslant c
$$

(W4) существует постоянная $\delta>0$, замкнутый интервал $\Lambda \subset \mathbb{R}$ и две последовательности положительных чисел $\sigma_{n} \rightarrow 0, M_{n} \rightarrow \infty$ при $n \rightarrow \infty$ такие, что

$$
\begin{array}{lll}
W(t, x) \geqslant-\delta|x|^{2} & \text { для всех } & (t, x) \in \Lambda \times B_{\rho}(0), \\
W(t, x) \geqslant \sigma_{n}^{2} M_{n} & \text { для всех } \quad t \in \Lambda \text { и }|x|=\sigma_{n} .
\end{array}
$$

Сформулируем основные результаты настоящей работы.

ТеОрема 1. Пусть выполнены условия (L3), (W3), (W4). Тогда существует последовательность $\left(x_{k}\right)$ решений системы (1.1) такая, что $\left\|x_{k}\right\|_{L^{\infty}} \rightarrow 0$ при $k \rightarrow \infty$.

СлеДСтвиЕ 1. Пусть выполнены условия (L3), (W3) и следующее условие:

(W'4) найдется число $r_{0} \in \mathbb{R}$ и постоянная $\tau>0$ такие, что

$$
\liminf _{|x| \rightarrow 0} \inf _{t \in\left[r_{0}-\tau, r_{0}+\tau\right]} \frac{W(t, x)}{|x|^{2}}>-\infty, \quad \limsup _{|x| \rightarrow 0} \inf _{t \in\left[r_{0}-\tau, r_{0}+\tau\right]} \frac{W(t, x)}{|x|^{2}}=+\infty .
$$

Тогда существует последовательность $\left(x_{k}\right)$ решений системъ (1.1) такая, что $\left\|x_{k}\right\|_{L^{\infty}} \rightarrow 0$ npu $k \rightarrow \infty$.

Для удобства читателя приведем пример, иллюстрирующий наши основные результаты.

ПримеР 1. Найдутся функции $L$ и $W$, которые удовлетворяют условиям теоремы 1 , но не удовлетворяют соответствующим условиям из упомянутых выше статей. Например, пусть $L(t)=h(t) I_{N}$, где

$$
\begin{gathered}
h(t)= \begin{cases}\left(p^{2}+1\right)^{2}(t-p)+c_{0}, & p \leqslant t<p+\frac{1}{p^{2}+1}, \\
\left(p^{2}+1\right)^{2}+c_{0}, & p+\frac{1}{p^{2}+1} \leqslant t<p+\frac{p^{2}}{p^{2}+1}, \\
\left(p^{2}+1\right)^{2}(p+1-t)+c_{0}, & p+\frac{p^{2}}{p^{2}+1} \leqslant t<p+1,\end{cases} \\
W(t, x)= \begin{cases}|x|^{s} \sin \frac{1}{|x|^{\varepsilon}} \cos t, & 0<|x|<1, \\
0, & x=0 ;\end{cases}
\end{gathered}
$$

здесь $I_{N}$ - единичная $(N \times N)$-матрица, $p \in \mathbb{Z}, c_{0} \in \mathbb{R}, \varepsilon \in(0,1)$ и $s \in(1+\varepsilon, 2)$. 
Всюду далее в статье мы обозначаем через $c, c_{i}$ различные положительные постоянные, которые могут варьироваться от формулы к формуле и не имеют существенного значения.

Статья организована следующим образом. Некоторые предварительные результаты представлены в разделе 2. Раздел 3 посвящен доказательству наших основных результатов.

\section{2. ПРЕДВАРИТЕЛЬНЫЕ РЕЗУЛЬТАТЫ}

2.1. Дробное исчисление Лиувилля-Вейля. Дадим два определения [9].

ОпРЕДЕЛЕНиЕ 1. Левый и правый дробные интегралы Лиувилля-Вейля порядка $0<\alpha<1$ на всей оси $\mathbb{R}$ задаются как

$$
{ }_{-\infty} I_{t}^{\alpha} x(t):=\frac{1}{\Gamma(\alpha)} \int_{-\infty}^{t}(t-\xi)^{\alpha-1} x(\xi) d \xi, \quad{ }_{t} I_{\infty}^{\alpha}(t):=\frac{1}{\Gamma(\alpha)} \int_{t}^{\infty}(\xi-t)^{\alpha-1} x(\xi) d \xi .
$$

ОпРЕДЕЛЕНиЕ 2. Левая и правая дробные производные Лиувилля-Вейля порядка $0<\alpha<1$ на всей оси $\mathbb{R}$ задаются как

$$
{ }_{-\infty} D_{t}^{\alpha} x(t):=\frac{d}{d t}-\infty I_{t}^{1-\alpha} x(t), \quad{ }_{t} D_{\infty}^{\alpha} x(t):=-\frac{d}{d t}{ }_{t} I_{\infty}^{1-\alpha} x(t) .
$$

ЗАмечАниЕ 1. Как отмечается в работе [9], определение (2.1) можно записать в альтернативном виде

$$
\begin{aligned}
-\infty & D_{t}^{\alpha} x(t)=\frac{\alpha}{\Gamma(1-\alpha)} \int_{0}^{\infty} \frac{x(t)-x(t-\xi)}{\xi^{\alpha+1}} d \xi \\
{ }_{t} D_{\infty}^{\alpha} x(t) & =\frac{\alpha}{\Gamma(1-\alpha)} \int_{0}^{\infty} \frac{x(t)-x(t+\xi)}{\xi^{\alpha+1}} d \xi .
\end{aligned}
$$

Напомним, что фурье-преобразование $\hat{x}(z)$ функции $x(t)$ определяется как

$$
\hat{x}(z)=\int_{-\infty}^{\infty} e^{-i t \cdot z} x(t) d t .
$$

Свойства фурье-преобразования дробных интегралов и дробных дифференциальных операторов были установлены в работе [9]:

$$
\begin{aligned}
& \widehat{-\infty I_{t}^{\alpha} x}(t)(z):=(i z)^{-\alpha} \hat{x}(z), \quad \widehat{{ }_{t} \alpha(t)}(z):=(-i z)^{-\alpha} \hat{x}(z), \\
& \left.\widehat{D_{t}^{\alpha} x}(t)(z):=(i z)^{\alpha} \hat{x}(z), \quad{ }_{t} \widehat{D_{\infty}^{\alpha} x(t}\right)(z):=(-i z)^{\alpha} \hat{x}(z) \text {. }
\end{aligned}
$$

2.2. Пространства дробных производных. Для каждого $\alpha>0$ полунорма и норма задаются как

$$
|x|_{I_{-\infty}^{\alpha}}:=\left\|{ }_{-\infty} D_{t}^{\alpha} x\right\|_{L^{2}}, \quad\|x\|_{I_{-\infty}^{\alpha}}:=\left(\|x\|_{L^{2}}^{2}+|x|_{I_{-\infty}^{\alpha}}^{2}\right)^{1 / 2} .
$$

Пусть пространство $I_{-\infty}^{\alpha}(\mathbb{R})$ есть пополнение пространства $C_{0}^{\infty}(\mathbb{R})$ по норме $\|\cdot\|_{I_{-\infty}^{\alpha}}$,

$$
I_{-\infty}^{\alpha}(\mathbb{R})=\overline{C_{0}^{\infty}(\mathbb{R})}\|\cdot\|_{I_{-\infty}^{\alpha}}
$$


В работе [9] для $0<\alpha<1$ в терминах фурье-преобразования были заданы полунорма $|x|_{\alpha}=\left\||z|^{\alpha} \hat{x}\right\|_{L^{2}}$ и норма $\|x\|_{\alpha}=\left(\|x\|_{L^{2}}^{2}+|x|_{\alpha}^{2}\right)^{1 / 2}$ и определено дробное пространство Соболева $H^{\alpha}(\mathbb{R})$,

$$
H^{\alpha}(\mathbb{R}):=\overline{C_{0}^{\infty}(\mathbb{R})}\|\cdot\|_{\alpha} .
$$

Заметим, что функция $x \in L^{2}(\mathbb{R})$ принадлежит пространству $I_{-\infty}^{\alpha}(\mathbb{R})$, если и только если $|z|^{\alpha} \hat{x} \in L^{2}(\mathbb{R})$. В частности, $|x|_{I_{-\infty}^{\alpha}}=\left\||z|^{\alpha} \hat{x}\right\|_{L^{2}(\mathbb{R})}$. Следовательно, $H^{\alpha}(\mathbb{R})$ и $I_{-\infty}^{\alpha}(\mathbb{R})$ - эквивалентные пространства с эквивалентными полунормой и нормой (см. работу [9]). Аналогично пространству $I_{-\infty}^{\alpha}(\mathbb{R})$ в работе [9] было введено пространство $I_{\infty}^{\alpha}(\mathbb{R})$. Пусть полунорма и норма заданы как

$$
|x|_{I_{\infty}^{\alpha}}:=\left\|_{t} D_{\infty}^{\alpha}\right\|_{L^{2}(\mathbb{R})}, \quad\|x\|_{I_{\infty}^{\alpha}}:=\left(\|x\|_{L^{2}}^{2}+|x|_{I_{\infty}^{\alpha}}^{2}\right)^{1 / 2},
$$

положим

$$
I_{\infty}^{\alpha}(\mathbb{R})={\overline{C_{0}^{\infty}(\mathbb{R})}}^{\|\cdot\|_{I_{\infty}^{\alpha}}} .
$$

При этом $I_{\infty}^{\alpha}(\mathbb{R})$ и $I_{-\infty}^{\alpha}(\mathbb{R})$ являются эквивалентами пространствами с эквивалентными полунормой и нормой.

Обозначим через $C(\mathbb{R})$ пространство непрерывных на $\mathbb{R}$ функций.

ЛЕмма 1 [9]. Если $\alpha>1 / 2$, то $H^{\alpha}(\mathbb{R}) \subset C(\mathbb{R})$ и существует постоянная $с=c_{\alpha}$ такая, что

$$
\|x\|_{L^{\infty}}=\sup _{t \in \mathbb{R}}|x(t)| \leqslant c\|x\|_{H^{\alpha}(\mathbb{R})} .
$$

ЗАмЕчАниЕ 2 . Если $x \in H^{\alpha}(\mathbb{R})$, то $x \in L^{q}(\mathbb{R})$ при любых $q \in[2, \infty]$, поскольку

$$
\int_{\mathbb{R}}|x(t)|^{q} d t \leqslant\|x\|_{L^{\infty}}^{q-2}\|x\|_{L^{2}}^{2}
$$

ЗАмечАниЕ 3. Рассмотрим следующую гамильтонову систему:

$$
-{ }_{t} D_{\infty}^{\alpha}\left({ }_{-\infty} D_{t}^{\alpha} x(t)\right)-\widehat{L}(t) x+\nabla \widehat{W}(t, x)=0, \quad x \in H^{\alpha}\left(\mathbb{R}, \mathbb{R}^{N}\right)
$$

где $\widehat{L}(t)=L(t)+a_{0} I_{N}$ и $\widehat{W}(t, x)=W(t, x)+\frac{a_{0}}{2}|x|^{2}$. Тогда система (2.4) эквивалентна (1.1) и легко проверить, что предположения (L3) и (W3), (W4) остаются выполненными для $\widehat{L}$ и $\widehat{W}$, если они выполнены для $L$ и $W$. На основании этого всюду далее мы полагаем без потери общности, что $l(t) \geqslant 1$ при всех $t \in \mathbb{R}$ и $\int_{\mathbb{R}} \frac{d t}{l(t)}<\infty$.

Учитывая замечание 3, рассмотрим пространство

$$
X^{\alpha}=\left\{x \in H^{\alpha}(\mathbb{R}): \int_{\mathbb{R}}\left(\left.\left.\right|_{-\infty} D_{t}^{\alpha} x(t)\right|^{2}+L(t) x(t) \cdot x(t)\right) d t<\infty\right\} .
$$

Оно является рефлексивным и сепарабельным гильбертовым пространством со скалярным произведением

$$
(x, y)_{X^{\alpha}}=\int_{\mathbb{R}}\left({ }_{-\infty} D_{t}^{\alpha} x(t) \cdot{ }_{-\infty} D_{t}^{\alpha} y(t)+L(t) x(t) \cdot y(t)\right) d t,
$$

и соответствующей нормой $\|x\|_{X^{\alpha}}=\sqrt{(x, x)_{X^{\alpha}}}$. 
ЛЕмма 2 [9]. Если бункиия L удовлетворяет условию (L3), то пространство $X^{\alpha}$ есть непрерывное вложение в $H^{\alpha}(\mathbb{R})$.

Мы докажем следующее утверждение.

ЛЕмма 3. Если функиия $L$ удовлетворяет условию (L3), то пространство $X^{\alpha}$ есть непрерывное вложение в $L^{1}$.

ДокАзАтЕльство. В силу условия (L3) и неравенства Гёльдера для всех $x \in X^{\alpha}$

$$
\begin{aligned}
\int_{\mathbb{R}}|x| d t & =\int_{\mathbb{R}}\left|L^{-1 / 2}(t) L^{1 / 2}(t) x\right| d t \leqslant \int_{\mathbb{R}} \frac{1}{\sqrt{l(t)}}\left|L^{1 / 2}(t) x\right| d t \leqslant \\
& \leqslant\left(\int_{\mathbb{R}} \frac{1}{l(t)} d t\right)^{1 / 2}\left(\int_{\mathbb{R}} L(t) x \cdot x d t\right)^{1 / 2} \leqslant\left(\int_{\mathbb{R}} \frac{1}{l(t)} d t\right)^{1 / 2}\|x\|_{X^{\alpha}} .
\end{aligned}
$$

Лемма 4. Если функиия L удовлетворяет условию (L3), то пространство $X^{\alpha}$ есть компактное вложение в $L^{1}$.

ДокАЗАтЕЛьство. Пусть $\left(x_{n}\right) \subset X^{\alpha}$ - ограниченная последовательность такая, что $x_{n} \rightarrow x$ в $X^{\alpha}$ (стрелкой $\rightarrow$ обозначена слабая сходимость). Покажем, что $x_{n} \rightarrow x$ по норме $L^{1}$. В силу неравенства Гёльдера мы имеем

$$
\begin{aligned}
\int_{\mathbb{R}}\left|x_{n}-x\right| d t= & \int_{|t| \leqslant R}\left|x_{n}-x\right| d t+\int_{|t|>R}\left|x_{n}-x\right| d t \leqslant \\
\leqslant & 2 R\left(\int_{|t| \leqslant R}\left|x_{n}-x\right|^{2} d t\right)^{1 / 2}+\int_{|t|>R}\left|L^{-1 / 2}(t) L^{1 / 2}(t)\left(x_{n}-x\right)\right| d t \leqslant \\
\leqslant & 2 R\left(\int_{|t| \leqslant R}\left|x_{n}-x\right|^{2} d t\right)^{1 / 2}+\int_{|t|>R} \frac{1}{\sqrt{l(t)}}\left|L^{1 / 2}(t)\left(x_{n}-x\right)\right| d t \leqslant \\
\leqslant & 2 R\left(\int_{|t| \leqslant R}\left|x_{n}-x\right|^{2} d t\right)^{1 / 2}+ \\
& +\left(\int_{|t|>R} \frac{1}{l(t)} d t\right)^{1 / 2}\left(\int_{|t|>R} L(t)\left(x_{n}-x\right) \cdot\left(x_{n}-x\right) d t\right)^{1 / 2} \leqslant \\
\leqslant & 2 R\left(\int_{|t| \leqslant R}\left|x_{n}-x\right|^{2} d t\right)^{1 / 2}+\left(\int_{|t|>R} \frac{1}{l(t)} d t\right)^{1 / 2}\left\|x_{n}-x\right\|_{X^{\alpha}}
\end{aligned}
$$

где $R>0$. В силу условия (L3) и теоремы Соболева о вложении для любого $\varepsilon>0$ существует $R_{0}>0$ такое, что при $R>R_{0}$ мы имеем

$$
\int_{\mathbb{R}}\left|x_{n}-x\right| d t \leqslant \varepsilon .
$$

\section{3. ДОКАЗАТЕЛЬСТВО ТЕОРЕМЫ 1 И СЛЕДСТВИЯ 1}

Для доказательства теоремы 1 и следствия 1 нам потребуется модифицировать функцию $W(t, x)$ при $x$, лежащих вне окрестности начала координат, чтобы получить всюду определенную функцию. Выберем постоянную $t_{0} \in(0, \rho / 2)$ и зададим обрезающую функцию $\chi \in C^{1}\left(\mathbb{R}^{+}, \mathbb{R}^{+}\right)$формулами

$$
\chi(t)=\left\{\begin{array}{l}
1, \quad 0 \leqslant t \leqslant t_{0}, \quad-\frac{2}{t_{0}} \leqslant \chi^{\prime}(t)<0 \text { при } t_{0}<t<2 t_{0} . \\
0, \quad t \geqslant 2 t_{0},
\end{array}\right.
$$


Пусть $\widetilde{W}(t, x)=\chi(|x|) W(t, x)$ для $(t, x) \in \mathbb{R} \times \mathbb{R}^{N}$. Из определения (3.1) и условия (W3) для всех $(t, x) \in \mathbb{R} \times \mathbb{R}^{N}$ мы имеем

$$
|\widetilde{W}(t, x)| \leqslant c_{1}|x|, \quad|\nabla \widetilde{W}(t, x)| \leqslant c_{2} .
$$

Теперь рассмотрим следующую модифицированную гамильтонову систему дробного порядка:

$$
-{ }_{t} D_{\infty}^{\alpha}\left({ }_{-\infty} D_{t}^{\alpha} x(t)\right)-L(t) x(t)+\nabla \widetilde{W}(t, x(t))=0, \quad x \in H^{\alpha}\left(\mathbb{R}, \mathbb{R}^{N}\right) .
$$

Зададим функционал $I: X^{\alpha} \rightarrow \mathbb{R}$, ассоциированный с системой $(3.3)$, как

$$
\begin{aligned}
I(x) & =\int_{\mathbb{R}}\left(\left.\left.\frac{1}{2}\right|_{-\infty} D_{t}^{\alpha} x(t)\right|^{2}+\frac{1}{2} L(t) x(t) \cdot x(t)-\widetilde{W}(t, x(t))\right) d t= \\
& =\frac{1}{2}\|x\|_{X^{\alpha}}^{2}-\int_{\mathbb{R}} \widetilde{W}(t, x(t)) d t .
\end{aligned}
$$

Тогда, учитывая условия (L3), (W3) и неравенства (3.2), заключаем, что $I$ есть непрерывный, дифференцируемый по Фреше функционал на $X^{\alpha}$, т. е. $I \in C^{1}\left(X^{\alpha}, \mathbb{R}\right)$. Кроме того, мы имеем для всех $x, y \in X^{\alpha}$

$$
I^{\prime}(x)(y)=\int_{\mathbb{R}}\left({ }_{-\infty} D_{t}^{\alpha} x(t) \cdot{ }_{-\infty} D_{t}^{\alpha} y(t)+L(t) x(t) \cdot y(t)-\nabla \widetilde{W}(t, x(t)) \cdot y(t)\right) d t .
$$

Согласно работе [9], чтобы найти решения задачи (3.3), достаточно найти критические точки функционала $I$. С этой целью напомним некоторые определения и результаты [10], [2].

ОПРЕДЕЛЕНИЕ 3. Пусть $E$ - действительное банахово пространство и функционал $\phi \in C^{1}(E, \mathbb{R})$.

- Говорят, что $\phi$ удовлетворяет условию (PS), если любая последовательность $\left(x_{k}\right) \subset E$, для которой последовательность $\left(\phi\left(x_{k}\right)\right)$ ограничена и $\phi^{\prime}\left(x_{k}\right) \rightarrow 0$ при $k \rightarrow+\infty$, содержит подпоследовательность, сходящуюся по норме $E$.

- Зададим множество

$\Sigma=\{A \subset E \backslash\{0\}: A$ замкнуто и симметрично относительно начала координат $\}$.

Для $A \in \Sigma$ будем говорить, что род множества $A$ есть $n$ (и писать $\kappa(A)=n$ ), если существует нечетное отображение $\varphi \in C\left(A, \mathbb{R}^{n} \backslash\{0\}\right)$ и $n$ есть минимальное целое число с этим свойством.

Положим $\Sigma_{k}=\{A \in \Sigma: \kappa(A) \geqslant k\}$.

ЛЕмма 5 [10]. Пусть $\phi-$ четный дифферениируемый функиионал на $E$ и $\phi(0)=0$. Предположим, что ф удовлетворяет условию (PS), ограничен снизу и для каждого $k \in \mathbb{N}$ найдется множество $A_{k} \in \Sigma_{k}$ такое, что $\sup _{x \in A_{k}} \phi(x)<0$. Тогда выполнено одно из следующих двух условий: либо существует последовательность критических точек $\left(x_{k}\right)$ такая, что $\phi\left(x_{k}\right)<0 u \lim _{k \rightarrow \infty} x_{k}=0$; либо существуют две последовательности критических точек $\left(x_{k}\right)$ u $\left(y_{k}\right)$ такие, что $\phi\left(x_{k}\right)=0, x_{k} \neq 0$, $\lim _{k \rightarrow \infty} x_{k}=0$ и $\phi\left(y_{k}\right)<0, \lim _{k \rightarrow \infty} \phi\left(y_{k}\right)=0,\left(y_{k}\right)$ сходится к ненулевому пределу. 
ЛЕмма 6. Если выполнены условия (L3), (W3), то функционал I ограничен снизу и удовлетворяет условию (PS).

ДоКАЗАТЕЛЬСТВо. В силу условия (W3), соотношений (2.5), (3.2) и неравенства Гёльдера мы имеем для всех $x \in X^{\alpha}$

$$
I(x) \geqslant \frac{1}{2}\|x\|_{X^{\alpha}}^{2}-c_{3} \int_{\mathbb{R}}|x| d t \geqslant \frac{1}{2}\|x\|_{X^{\alpha}}^{2}-c_{3}\left(\int_{\mathbb{R}} \frac{1}{l(t)} d t\right)^{1 / 2}\|x\|_{X^{\alpha}} .
$$

Отсюда следует, что $I$ ограничен снизу. Далее, если последовательность $\left(x_{n}\right) \subset X^{\alpha}$ взята из условия (PS), то в силу (3.6) мы имеем

$$
c_{4} \geqslant \frac{1}{2}\left\|x_{n}\right\|_{X^{\alpha}}^{2}-c_{5}\left(\int_{\mathbb{R}} \frac{1}{l(t)} d t\right)^{1 / 2}\|x\|_{X^{\alpha}} .
$$

Это влечет, что $\left(x_{n}\right)$ ограничена по норме $X^{\alpha}$. Следовательно, существует подпоследовательность $\left(x_{n_{k}}\right)$ такая, что $x_{n_{k}} \rightarrow x_{0}$ при $k \rightarrow \infty$ для некоторого $x_{0} \in X^{\alpha}$. По лемме 4 мы имеем, что $x_{n_{k}} \rightarrow x_{0}$ в $L^{1}$ при $k \rightarrow \infty$. Вместе с неравенствами (3.2) это дает

$$
\left|\int_{\mathbb{R}}\left(\nabla \widetilde{W}\left(t, x_{n_{k}}\right)-\nabla \widetilde{W}\left(t, x_{n_{k}}\right)\right) \cdot\left(x_{n_{k}}-x_{0}\right) d t\right| \leqslant c_{6} \int_{\mathbb{R}}\left|x_{n_{k}}-x_{0}\right| d t \rightarrow 0 \text { при } k \rightarrow \infty .
$$

Поскольку последовательность $\left(x_{n}\right) \subset X^{\alpha}$ взята из условия (PS) и ограничена,

$$
\left(I^{\prime}\left(x_{n_{k}}\right)-I^{\prime}\left(x_{0}\right)\right)\left(x_{n_{k}}-x_{0}\right) \rightarrow 0 \quad \text { при } \quad k \rightarrow \infty .
$$

Комбинируя соотношения (3.5), (3.7) и (3.8), получаем

$$
\begin{aligned}
& \left\|x_{n_{k}}-x_{0}\right\|_{X^{\alpha}}^{2}= \\
& \quad=\left(I^{\prime}\left(x_{n_{k}}\right)-I^{\prime}\left(x_{0}\right)\right)\left(x_{n_{k}}-x_{0}\right)+\int_{\mathbb{R}}\left(\nabla \widetilde{W}\left(t, x_{n_{k}}\right)-\nabla \widetilde{W}\left(t, x_{0}\right)\right) \cdot\left(x_{n_{k}}-x_{0}\right) d t \rightarrow 0 .
\end{aligned}
$$

ДОКАЗАТЕЛЬСТВо ТЕОРЕМЫ 1. Пусть выполнены условия (L3) и (W4), тогда для каждого $k \in \mathbb{N}$ найдется множество $A_{k} \in \Sigma_{k}$ с $\kappa\left(A_{k}\right)=k$ такое, что $\sup _{x \in A_{k}} I(x)<0$. В самом деле, следуя идеям решения эллиптических задач из работы [10], мы имеем следующее.

Пусть $\eta$ - длина замкнутого интервала $\Lambda$ из условия (W4). Для любого фиксированного $k \in \mathbb{N}$ разобьем $\Lambda$ на $k$ одинаковых замкнутых интервалов $\Lambda_{i}, 1 \leqslant i \leqslant k$, длины $\eta / k$. Пусть $s_{i}$ - центр интервала $\Lambda_{i}$ и $\Omega_{i}$ - замкнутый интервал с центром в $s_{i}$ длины $\eta / 2 k, 1 \leqslant i \leqslant k$. Выберем функцию $\xi \in C_{0}^{\infty}\left(\mathbb{R}, \mathbb{R}^{N}\right)$ такую, что

$$
|\xi(t)| \equiv \begin{cases}1, & t \in\left[-\frac{\eta}{4 k}, \frac{\eta}{4 k}\right] \\ 0, & t \in \mathbb{R} \backslash\left[\frac{\eta}{2 k}, \frac{\eta}{2 k}\right]\end{cases}
$$

и $|\xi(t)| \leqslant 1$ при всех $t \in \mathbb{R}$. Зададим функции $\xi_{i} \in C_{0}^{\infty}\left(\mathbb{R}, \mathbb{R}^{N}\right)$ как $\xi_{i}(t)=\xi\left(t-t_{i}\right)$ для всех $t \in \mathbb{R}$. Тогда мы имеем

$$
\begin{array}{llll}
\operatorname{supp} \xi_{i} \subset \Lambda_{i}, & \left|\xi_{i}(t)\right|=1 & \text { при всех } & t \in \Omega_{i}, \\
& \left|\xi_{i}(t)\right| \leqslant 1 & \text { при всех } & t \in \mathbb{R} .
\end{array}
$$


Пусть

$$
\Theta_{k} \equiv\left\{\left(l_{1}, l_{2}, \ldots, l_{k}\right) \in \mathbb{R}^{k}: \max _{1 \leqslant i \leqslant k}\left|l_{i}\right|=1\right\}, \quad S_{k} \equiv\left\{\sum_{i=1}^{k} l_{i} \xi_{i}:\left(l_{1}, l_{2}, \ldots, l_{k}\right) \in \Theta_{k}\right\} .
$$

Тогда множество $\Theta_{k}$ с помощью нечетного отображения можно гомеоморфно перевести в единичную сферу в $\mathbb{R}^{k}$. Следовательно, $\kappa\left(\Theta_{k}\right)=k$. Теперь зададим нечетное гомеоморфное отображение $\psi: \Theta_{k} \rightarrow S_{k}$ как

$$
\psi\left(l_{1}, l_{2}, \ldots, l_{k}\right)=\sum_{i}^{k} l_{i} \xi_{i}, \quad\left(l_{1}, l_{2}, \ldots, l_{k}\right) \in \Theta_{k} .
$$

Тогда $\kappa\left(S_{k}\right)=\kappa\left(\Theta_{k}\right)=k$. Очевидно, что множество $S_{k}$ компактно, следовательно, существует постоянная $\lambda_{k}>0$ такая, что $\|x\|_{X^{\alpha}} \leqslant \lambda_{k}$ для всех $x \in S_{k}$.

Далее, для любого $s \in\left(0, t_{0}\right)$ и для любого $x=\sum_{i=1}^{k} l_{i} \xi_{i} \in S_{k}$ в силу соотношений (3.1), (3.4) и (3.9) мы имеем

$$
\begin{aligned}
I(s x) & =\frac{1}{2}\|s x\|_{X^{\alpha}}^{2}-\int_{\mathbb{R}} \widetilde{W}\left(t, s \sum_{i=1}^{k} l_{i} \xi_{i}\right) d t= \\
& =\frac{s^{2}}{2}\|x\|_{X^{\alpha}}^{2}-\sum_{i=1}^{k} \int_{\Lambda_{i}} \widetilde{W}\left(t, s l_{i} \xi_{i}\right) d t=\frac{s^{2}}{2}\|x\|_{X^{\alpha}}^{2}-\sum_{i=1}^{k} \int_{\Lambda_{i}} W\left(t, s l_{i} \xi_{i}\right) d t .
\end{aligned}
$$

По определению множества $\Theta_{k}$ для любого $x=\sum_{i}^{k} l_{i} \xi_{i} \in S_{k}$ существует некоторое целое число $i_{x}, 1 \leqslant i_{x} \leqslant k$, такое, что $\left|l_{i_{x}}\right|=1$. Тогда по условию (W4) с учетом соотношения (3.9) и определения $\Theta_{k}$ получаем, что

$$
\begin{aligned}
& \sum_{i=1}^{k} \int_{\Lambda_{i}} W\left(t, s l_{i} \xi_{i}\right) d t= \\
& \quad=\int_{\Omega_{i_{x}}} W\left(t, s l_{i} \xi_{i}\right) d t+\int_{\Theta_{i_{x}} \backslash \Omega_{i_{x}}} W\left(t, s l_{i_{x}} \xi_{i_{x}}\right) d t+\sum_{i \neq i_{x}} \int_{\Lambda_{i}} W\left(t, s l_{i} \xi_{i}\right) d t \geqslant \\
& \quad \geqslant \int_{\Omega_{i_{x}}} W\left(t, s l_{i} \xi_{i}\right) d t-\delta \eta s^{2} .
\end{aligned}
$$

Для $\sigma_{n} \in\left(0, t_{0}\right)$, комбинируя условие (W4) и формулы $(3.1),(3.9)-(3.11)$, мы имеем

$$
I\left(\sigma_{n} x\right)=\frac{\lambda_{k} \sigma_{n}^{2}}{2}+\delta \eta \sigma_{n}^{2}-\int_{\Omega_{i_{x}}} \widetilde{W}\left(t, \sigma_{n} l_{i_{x}} \xi_{i_{x}}\right) d t=\sigma_{n}^{2}\left(\frac{\lambda_{k}}{2}+\delta \eta-\frac{M_{n} \eta}{2 k}\right) .
$$

Здесь мы использовали тот факт, что $\left|\sigma_{n} l_{i_{x}} \xi_{i_{x}}(t)\right| \equiv \sigma_{n}$ при $t \in \Omega_{i_{x}}$. Поскольку $M_{n} \rightarrow \infty$ при $n \rightarrow \infty$, можно выбрать $n_{k} \in \mathbb{N}$ так, что $M_{n_{k}} \geqslant 2 k \lambda_{k} / \eta+2 k \delta$. Из (3.12) получаем оценку

$$
I\left(\sigma_{n} x\right) \leqslant-\frac{\lambda_{k} \sigma_{n_{k}}^{2}}{2}
$$

Положим $A_{k}=\left\{\sigma_{n_{k}} x: x \in S_{k}\right\}$. Тогда $\kappa\left(A_{k}\right)=\kappa\left(S_{k}\right)=k$ и $\sup _{x \in A_{k}} I(x)<0$. Следовательно, по лемме 4 найдется последовательность $\left(x_{k}\right)$ нетривиальных критических 
точек функционала $I$ такая, что $I\left(x_{k}\right) \leqslant 0$ при всех $k \in \mathbb{N}$ и $x_{k} \rightarrow 0$ в $X^{\alpha}$ при $k \rightarrow \infty$. С учетом (2.3) отсюда следует, что $\left\|x_{k}\right\|_{L^{\infty}} \rightarrow 0$ при $k \rightarrow \infty$. Поскольку $\left(x_{k}\right)$ - это последовательность решений задачи (3.3), для достаточно больших $k$ функции $x_{k}$ являются решениями задачи (1.1).

ДоКАЗАТЕЛЬСТво СЛЕДСТВИЯ 1. По условию $\left(\mathrm{W}^{\prime} 4\right)$ найдется постоянная $\delta>0$ и две последовательности положительных чисел $\sigma_{n} \rightarrow 0, M_{n} \rightarrow \infty$ при $n \rightarrow \infty$ такие, что

$$
\begin{array}{lll}
W(t, x) \geqslant-\delta|x|^{2} & \text { при всех } & (t, x) \in\left[r_{0}-\tau, r_{0}+\tau\right] \times B_{\rho}(0), \\
W(t, x) \geqslant \sigma_{n}^{2} M_{n} & \text { при всех } & t \in\left[r_{0}-\tau, r_{0}+\tau\right] \text { и }|x|=\sigma_{n},
\end{array}
$$

откуда следует, что выполнено условие (W4). Простое применение теоремы 1 доказывает следствие 1.

Благодарности. Автор благодарен рецензенту за внимательное чтение статьи, критические замечания и полезные предложения, которые помогли улучшить качество изложения.

\section{Список литературы}

[1] A. Ambrosetti, P.H. Rabinowitz, "Dual variational methods in critical point theory and applications", J. Funct. Anal., 14:4 (1973), 349-381.

[2] P. H. Rabinowitz, Minimax Methods in Critical Point Theory with Applications to Differential Equations, CBMS Regional Conference Series in Mathematics, 65, AMS, Providence, RI, 1986.

[3] A. Ambrosetti, V. C. Zelati, "Multiple homoclinic orbits for a class of conservative systems", Rend. Semin. Mat. Univ. Padova, 89 (1993), 177-194.

[4] Y.H. Ding, "Existence and multiplicity results for homoclinic solutions to a class of Hamiltonian systems", Nonlinear Anal.: Theor. Methods Appl., 25:11 (1995), 1095-1113.

[5] W. Omana, M. Willem, "Homoclinic orbits for a class of Hamiltonian systems", Differ. Integral Equ., 5:5 (1992), 1115-1120.

[6] A. A. Kilbas, H. M. Srivastava, J. J. Trujillo, Theory and Applications of Fractional Differential Equations, North-Holland Mathematics Studies, 204, Elsevier, Amsterdam, 2006.

[7] K. S. Miller, B. Ross, An Introduction to the Fractional Calculus And Fractional Differential Equations, Wiley and Sons, New York, 1993.

[8] F. Jiao, Y. Zhou, "Existence results for fractional boundary value problem via critical point theory", Internat. J. Bifurcation Chaos, 22:4 (2012), 1250086, 17 pp.

[9] C. Torres, "Existence of solution for fractional Hamiltonian systems", Electron. J. Differ. Equ., 2013:259 (2013), 1-12.

[10] R. Kajikiya, "A critical point theorem related to the symmetric mountain pass lemma and its applications to elliptic equations", J. Funct. Anal., 225:2 (2005), 352-370.

Поступила в редакцию 22.03.2017, после доработки 25.08.2017 